\title{
EFFECT OF FLUCTUATIONS ON THE THERMAL CONDUCTIVITY OF HE I NEAR $T_{\lambda}{ }^{*}$
}

\author{
H. GOULD and V.K. WONG \\ Department of Physics, University of Michigan, Ann Arbor, Michigan 48104, USA
}

Received 13 February 1970

\begin{abstract}
A microscopic calculation of the contribution of order-parameter fluctuations to the thermal conductivity of liquid helium I near $T_{\lambda}$ is shown to give results in agreement with experiments and the dynamic scaling hypothesis.
\end{abstract}

The anomalous increase in the thermal conductivity $[1,2]$ in helium I near $T_{\lambda}$ has been successfully analyzed in terms of the dynamic scaling hypothesis [3,4], mode-mode coupling [5] and the generalized Landau theory [6]. The above theories are phenomenological in nature.

Aslamazov and Larkin [7] have calculated microscopically the contribution of the order-parameter fluctuations to the electric conductivity of a normal metal above the superconducting transition in the classical range. Tsuzuki [8] has extended the calculation to the critical range.

The purpose of this letter, which is the analog of refs. 7 and 8 for the superfluid transition, is to report a microscopic calculation of the contribution of order-parameter fluctuations to the thermal conductivity of helium I near $T_{\lambda}$.

Recently the authors [9] have been able to reformulate the theory of Patashinskli and Pokrovskii [10] to describe consistently the order-parameter fluctuations in the experimentally accessible temperature range near $T_{\lambda}$. The order parameter appropriate to the superfluid transition is the Bose field operator $\psi(r, t)$, and hence orderparameter fluctuations are described by the fluctuation propagator $G\left(l l l^{\prime}\right)=-i\left\langle T\left\{\psi(l) \psi^{+}\left(l^{\prime}\right)\right\}\right\rangle$, $l=\eta, t_{l}$. The Fourier transform can be written as $G^{-l}\left(k, \omega \pm i 0^{+}\right)=\omega-W(k, \omega) \mp i \frac{1}{2} \Gamma(k, \omega)$. The microscopic theory gives the following results for small $k$ and $\omega$ near $T_{\lambda}$ :

$W(k, \omega)=W(k, 0) \approx \begin{cases}A_{1} k^{2}+\hat{\eta} & 0<k<k_{1} \\ A_{0} k^{\frac{3}{2}}+\hat{\eta} & k_{1}<k<k_{0} \\ k^{2} / 2 m+\hat{\eta} & k_{0}<k\end{cases}$

* Supported in part by the National Science Foundation and the Horace H. Rackham School of Graduate Studies. and $\Gamma(k, \omega)_{0}=\gamma_{0} \omega_{2}$ where $A_{0}^{4}=(2 / 15 \pi)\left(T_{\lambda} V_{0}\right)^{2}$, $A_{1}^{3}=\left(T_{\lambda} V_{0}\right)^{2} / 432 \pi^{2} \hat{\eta}, \quad \hat{\eta}=\frac{3}{2} n V_{0} \tau, \tau=\left(T-T_{\lambda}\right) / T_{\lambda}$, $V_{0}=\int \mathrm{d} r V(r), V(r)$ is the interparticle potential, the momenta $k_{0}, k_{1}$ satisfy $A_{0} k_{0}^{\frac{3}{2}}=k_{0}^{2} / 2 m$ and $A_{1} k_{1}^{2}=A_{0} k_{1}^{\frac{3}{2}}$ respectively, and $\gamma_{0}$ is a constant. An essential feature of the present theory is the damping $\Gamma$ of the critical fluctuations, which was ignored in ref. 10. Since the damping is of the same order of magnitude as the real part, it is misleading to refer to helium near $T_{\lambda}$ as behaving like an Ideal gas of quasi-particles.

The thermal conductivity $\kappa$ can be found from eq. $(88 \mathrm{~b})$ in ref. 11 in-terms of the auto correlation function of $j^{\prime}=j^{q}+\lambda g$. We use the notation of ref. 11 and choose $\lambda=(p+T s) / n m$, which gives

$$
j^{\prime}=\left[T s(1)-\frac{T s}{n} n(1)+p(1)-p+T s\right] v(1)
$$

If we ignore the fluctuations of entropy and density, (2) reduces to $j^{\prime}=T s v$, and the contribution of order parameter fluctuations to $\kappa$ is

$\kappa^{\gamma}=\frac{1}{2} s^{2} \lim _{\omega \rightarrow 0}\left[\lim _{\rightarrow 0}\left\langle v_{x}(k, \omega) v_{x}(-k,-\omega)\right\rangle\right]$.

The correlation function $\langle v v\rangle$, which has the structure of a two-particle correlation function, is factorized into a product of two fluctuation propagators in analogy to refs. 7 and 8 . The corresponding integral for $\kappa^{\prime}$ is

$\kappa^{\prime}=\frac{1}{6} \frac{s^{2}}{m^{2}} \frac{1}{\beta} \sum_{n} \int \frac{\mathrm{d} p}{(2 \pi)^{3}} p^{2} Q^{2} G\left(p, \omega_{n}+\mathrm{i0} 0^{+}\right) G\left(p, \omega_{n}\right)$.

The vertex function $Q$ is determined by the Ward identity

$$
k Q=-m \nabla_{k} G^{-1}(k, 0)=2 m A_{1} k .
$$


Integrals similar to (4) are performed in refs. 7 and 8 , and we find

$$
\kappa^{\prime}=(32 \pi)^{-1}\left(T_{\lambda} s / n\right)^{2} \gamma_{0}\left(\hat{\eta} A_{1}\right)^{-\frac{1}{2}} \propto \tau^{-\frac{1}{3}} \text {. }
$$

It is seen that the contribution of order-parameter fluctuations to $\kappa$ diverges as $\tau^{-\frac{1}{3}}$ consistent with the phenomenological theories [3-6] except for the absence of logarithmic factors. The extended dynamical scaling [4] and mode-mode coupling [5] theories give a critical temperature dependence for $\kappa^{\prime}$ proportional to $\tau^{-\frac{1}{3}} C_{p}^{+} / C_{p}^{-\frac{1}{3}}$ where $C_{p}^{+}$and $C_{p}^{-}$is the specific heat at constant pressure above and below $T_{\lambda}$ respectively*. The factors of $C_{p}$ arise in ref. 5 from the normalization of the entropy fluctuations which have been neglected here. The origin of $C_{p}$ factors in the other theories can also be considered as arising from the entropy fluctuations. The pro* Refs. 3 and 6 obtain $\tau^{-\frac{1}{3}} C_{p}^{-\frac{1}{2}}$. blem of including the contribution of entropy fluctuations to the thermal conductivity into a consistent, microscopic theory is discussed in ref. 9.

\section{References}

1. G. Ahlers, Phys. Rev. Letters 21 (1968) 1159.

2. M. Archibald, J. M. Mochel and L. Weaver, Phys. Rev, Letters 21 (1968) 1156.

3. R. A. Ferrell et al., Ann. Phys. (NY) 47 (1968) 565.

4. B. I. Halperin and P. C. Hohenberg, Phys. Rev. 177 (1969) 952.

5. J. Swift and L. P. Kadanoff, Ann. Phys. 50 (1968) 312.

6. D. Stauffer and V.K. Wong, to be published.

7. L. G. Aslamazov and A. I. Larkin, Soviet Phys. Solid State 10 (1968) 875.

8. T. Tsuzuki, Prog. Theoret. Phys. (Kyoto) 41 (1969) 41.

9. H. Gould and V.K. Wong, to be published.

10. A. Z. Patashinskii and V. L. Pokrovskii, Soviet Phys. JETP 19 (1964) 677.

11. L. P. Kadanoff and P. C. Martin, Ann. Phys. (NY) 24 (1963) 419.

\title{
IONICITIES, EFFECTIVE CHARGES, DIELECTRIC CONSTANTS,
} AND ATOMIC POLARIZABILITIES OF III-V SEMICONDUCTORS

\author{
K. HÜBNER \\ Sektion Physik der Karl-Marx-Universität, Leipzig, DDR
}

Received 24 February 1970

Relative sharing times of a valence electron are used to calculate particular dielectric constant and polar-
izabilities of the constituent atoms from optical effective charges and dielectric constants of the compound.

The real chemical bond in III- $V$ compounts is usually desçribed as a superposition of an ionic structure $A_{\mathrm{II}}^{3+} \mathrm{B}^{3-}$ (probalility $w$ ) and a covalent structure $\mathrm{A}_{\mathrm{III}}^{\mathrm{I}} \mathrm{B}_{\mathrm{V}}^{\mathrm{I+}}$ (probability 1-w). For both the electrons in one of the four tetraedrical bonds follows:

$$
\Psi^{2}(1,2)=w \cdot \psi_{\text {ion }}^{2}(1,2)+(1-w) \cdot \psi_{\text {cov }}^{2}(1,2) .(1)
$$

Disregarding overlap (for the values of overlap integrals of III-V compounds see ref. 1 we put $\psi_{\text {cov }}(1,2)=\frac{1}{2} \sqrt{2}\left(\Phi_{I I}(1) \cdot \Phi_{V}(2)+\Phi_{I I}(2) \Phi_{V}(1)\right)$ for the covalent structure and $\psi_{\text {ion }}(1,2)=$ $=\Phi_{V}(1) \Phi_{V}(2)$ for the ionic structure, resp., ( $\Phi_{\mathrm{III}}, \Phi_{\mathrm{V}}$-normalized electronic wave functions of the III- and the V-atom, resp., in the crystal.).

Integrating (1) over variables of one electron we see that a valence electron spends $\frac{1}{2}(1 \mp w)$ of its time in the wave function of the III-atom and the $\mathrm{V}$-atom, resp. (These sharing times can be used for lending weight to atomic relativistic effects of constituents to predict the same effects of the compounds [1].) This means, the net number of valence electrons is $8(1 \mp w) / 2$ at the IIIatomic site and the $\mathrm{V}$-atomic site, resp., in the crystal. For $w=1 / 4$ the atoms posses their natural number of valence electrons; the bond is neutral (configuration $A_{\text {III }}^{O} B_{V}^{O}$ ).

Introducing different optical dielectric constants $\epsilon_{\mathrm{III}}$ and $\epsilon_{\mathrm{V}}$ for the III-atoms and $\mathrm{V}$-atoms, resp., in the crystal [2], one can deduce "macroscopic" charges $e \stackrel{\pi}{\pi} / \mathrm{V}=8(1 \mp w) / 2 \epsilon \mathrm{IIV} / \mathrm{V}$ from the "microscopic" net charges mentioned above.

Their difference produces the effective charge $e_{\mathrm{c}}^{*}$ defined by Callen [3], which splits the longitudinal and transverse optical frequencies.

$$
e_{\mathrm{c}}^{*}=\left(e_{\mathrm{V}}^{*}-e_{\mathrm{III}}^{*}\right) \cdot 1 / 2
$$

The sum of the "macroscopic" charges per bond 